\title{
Características anatômicas do plexo braquial de bicho-preguiça-de-coleira (Bradypus torquatus Illiger, 1811)
}

\author{
Gessica Ariane de Melo Cruz ${ }^{1}$ \\ Marta Adami $2 *$ \\ Vera Lúcia de Oliveira ${ }^{3}$ \\ ${ }^{1}$ Médica Veterinária, Salvador - BA, Brasil \\ ${ }^{2}$ Departamento de Anatomia Veterinária, Escola de Medicina Veterinária e Zootecnia \\ Universidade Federal da Bahia \\ Avenida Adhemar de Barros, 500, CEP 40170-110, Salvador - BA, Brasil \\ ${ }^{3}$ Reserva Zoobotânica Matinha, Comissão Executiva do Plano da Lavoura Cacaueira \\ Ilhéus - BA, Brasil \\ *Autor para correspondência \\ madami@ufba.br
}

Submetido em 29/11/2012

Aceito para publicação em 26/04/2013

\section{Resumo}

Oito cadáveres machos e fêmeas previamente formolizados de bicho-preguiça-de-coleira (Bradypus torquatus) foram utilizados para a identificação da origem, nervos e território de inervação do plexo braquial, visando o estabelecimento de um padrão anatômico para a espécie. O plexo braquial de bicho-preguiça-de-coleira derivou-se dos nervos espinhais $\mathrm{C} 7$ a C10 e T1 a T2, sendo a participação de T2 variável. Os nervos espinhais originaram os troncos cranial e caudal que se uniram e formaram um tronco comum que emitiu dois fascículos, a partir dos quais se originaram todos os nervos do plexo braquial, com exceção dos nervos peitorais, torácico longo e supraescapular, que surgiram antes da formação do tronco comum. A organização do plexo braquial em troncos e fascículos, com posterior origem dos nervos periféricos, indica a contribuição da maioria dos nervos espinhais relacionados na composição dos nervos periféricos e a possibilidade de comprometimento de grande parte do membro torácico em situações de traumas ou lesões.

Palavras-chave: Membro torácico; Sistema nervoso; Xenarthra

\section{Abstract}

Anatomical characteristics of the brachial plexus of the maned sloth (Bradypus torquatus Illiger, 1811). Eight male and female maned sloth (Bradypus torquatus) cadavers, previously fixed in formalin, were used to identify the origin of the brachial plexus, nerves and innervation territory in order to determine an anatomical pattern for this species. The plexus of B. torquatus was derived from the $\mathrm{C} 7$ to $\mathrm{C} 10$ and $\mathrm{T} 1$ to $\mathrm{T} 2$ spinal nerves, but the participation of T2 was variable. The spinal nerves gave origin to the cranial and caudal trunks, which joined to form a common trunk, from which two fascicles were formed. All the nerves from the brachial plexus were originated from these two fascicles, except the thoracic, long pectoral and suprascapular nerves, which arose before the formation of the common trunk. The organization of the brachial plexus into trunks and fascicles, and subsequent origin of peripheral nerves, demonstrates that most of the spinal nerves contribute to the composition of the peripheral nerves and the possibility that lesions or traumatic injuries would damage most of the thoracic member.

Key words: Forelimb; Nervous system; Xenarthra 


\section{Introdução}

O bicho-preguiça-de-coleira (Bradypus torquatus) é um mamífero da ordem Xenarthra, possui dieta estritamente folívora, baixo nível de atividade, movimentos vagarosos (CHIARELLO, 1998; FERNANDEZ; MIRANDA, 2007) e só desce ao chão para urinar e defecar (MENDEL, 1985). Os animais desta espécie vivem na Mata Atlântica do sul de Sergipe ao norte do Rio de Janeiro, sendo considerado uma das espécies mais raras da sua ordem, sendo que o gênero Bradypus está classificado como vulnerável pela International Union for Conservation of Nature (IUCN), resultado do processo de destruição e fragmentação de seu hábitat, de uma reprodução sazonal e do impacto da caça (CHIARELLO et al., 2011). As preguiças raramente assumem uma postura ereta, no solo se movimentam com os membros distantes do corpo denotando falta de equilíbrio, lentidão e dificuldade, além do alto gasto de energia (MENDEL, 1985).

Nos mamíferos domésticos, o número de segmentos medulares cervicais corresponde a oito, apesar de todos apresentarem sete vertebrais cervicais. Dessa maneira, o primeiro nervo espinhal cervical emerge cranialmente à vértebra respectiva e o último - oitavo nervo cervical caudalmente a sétima vértebra cervical (GETTY et al., 1986). Alguns animais silvestres apresentam variação no número de vértebras cervicais, podendo ser citados o peixe-boi (Trichechus sp.), preguiça de dois dedos (Choloepus sp.) e preguiça de três dedos (Bradypus). Em preguiça de dois-dedos, pode haver variações entre cinco ou seis vértebras (Choloepus hofmanni) e seis ou sete vértebras (Choloepus didactylus). Em preguiça de três-dedos (Bradypus sp.), esta variação ocorre entre oito ou nove vértebras cervicais (HAUTIER et al., 2010; VARELA-LASHERAS et al., 2011). Contudo, apesar do número variável de vértebras cervicais, a preguiça segue o mesmo padrão de surgimento do primeiro par de nervo espinhal cervical (C1) entre o occipital e o atlas e o último par de nervo espinhal cervical (C10), entre a última vértebra cervical (nona vértebra) e a primeira vértebra torácica (BIELIK, 1937).

O plexo braquial de animais silvestres tem sido amplamente estudado em espécies domésticas (MAGILTON et al., 1968) e silvestres como em primatas (MIZUNO, 1969; BOOTH et al., 1997), antílope japonês
(ATOJI et al., 1987), camelo bactriano (WANG, 1996), monotremados (KOIZUMI; SAKAI, 1997), capivara (FIORETTO et al., 2003), mocó (SANTANA et al., 2003), porco-espinho (AYDIN, 2003; 2004), chinchila (GAMBA et al., 2007), veado (MELO et al., 2007), cateto (MOURA et al., 2007), paca (SCAVONE et al., 2008), dentre outras espécies. Bielik (1937) estudou a constituição do plexo braquial em preguiça-debentinho (Bradypus trydactilus) e Amorim et al. (2003) descreveram a origem do plexo braquial em bichopreguiça-comum (Bradypus variegatus).

Objetivamos descrever o plexo braquial de bichopreguiça-de-coleira (Bradypus torquatus) em sua origem, composição, nervos derivados e território de inervação e assim estabelecer um modelo anatômico para a espécie, com a ampliação e divulgação de informações sobre a biologia de animais silvestres.

\section{Material e Métodos}

Foram utilizados oito animais, sendo quatro machos e quatro fêmeas, cedidos ao Departamento de Anatomia, Patologia e Clínicas Veterinárias da Escola de Medicina Veterinária e Zootecnia da Universidade Federal da Bahia (EMV-UFBA), pelo Centro de Estudos de Bradypus, localizado na Reserva Zoobotânica (Matinha) da Comissão Executiva do Plano da Lavoura Cacaueira (CEPLAC), Ilhéus, Bahia. A pesquisa foi autorizada pelo Sistema de Autorização e Informação em Biodiversidade (SISBIO) do Instituto Brasileiro do Meio Ambiente e dos Recursos Naturais Renováveis (IBAMA) com o n 18267-1 em 03/02/2009. Os animais já se encontravam fixados em solução de formol a $10 \%$ e, posteriormente, foram dissecados e realizada a identificação da origem, os nervos que compuseram o plexo braquial e o território de inervação. A terminologia adotada foi baseada no International Committee On Veterinary Gross Anatomical Nomenclature (2005).

\section{Resultados}

O bicho-preguiça-de-coleira (Bradypus torquatus) possui nove vértebras cervicais e dez pares de nervos espinhais cervicais, com a emergência do primeiro par 
de nervo espinhal cervical entre o occipital e o atlas e o último par (C10) entre a nona vértebra cervical e a primeira torácica. O plexo braquial derivou-se a partir do $7^{\circ}, 8^{\circ}, 9^{\circ}$ e $10^{\circ}$ segmentos medulares cervicais e $1^{\circ} \stackrel{\mathrm{e}}{ }$ 2 o torácicos, que corresponderam aos respectivos nervos espinhais relacionados: $\mathrm{C} 7$ a $\mathrm{C} 10$ e T1 a T2. Em 4 casos houve contribuição de $\mathrm{T} 2$ que participou na formação do tronco caudal com uma anastomose para T1 (Figura 1).

Os nervos espinhais originaram dois troncos: cranial e caudal que se uniram formando um tronco comum que emitiu dois fascículos, a partir dos quais se originaram quase todos os nervos do plexo braquial, com exceção do nervo supraescapular (dois animais), que se originou apenas de $\mathrm{C} 7$; o nervo torácico longo (um animal) de $\mathrm{C} 9$ e os nervos peitorais que derivaram somente do tronco caudal (Figura 2).

O nervo supraescapular (NSP) originou-se do tronco cranial ou apenas de C7 e supriu os músculos supraespinhoso e infraespinhoso. O nervo torácico longo (NTL) originou-se somente de C9 e seguiu para o músculo serrátil ventral do tórax. Os nervos peitorais (Nn.P) originaram-se do tronco caudal ou do fascículo ventral e ramificaram-se nos músculos peitorais.

FIGURA 1: Desenho esquemático de plexo braquial de bicho-preguiça-de-coleira (Bradypus torquatus). NM-MC: nervo medianomusculocutâneo; NR: nervo radial; NA: nervo axilar; NU: nervo ulnar; NSB: nervo subescapular; NSP: nervo supraescapular; NTL: nervo torácico longo; NTD: nervo toracodorsal; NCMA: nervo cutâneo medial do antebraço NnP: nervos peitorais; rNM-MC: ramo muscular proximal do nervo mediano-musculocutâneo; NI: nervo intercostal. C6 a C9: sexta a nona vértebras cervicais; T1 a T3: primeira a terceira vértebras torácicas; C7 a C10 em negrito: sétimo a décimo nervos espinhais cervicais; T1 e T2 em negrito: primeiro e segundo nervos espinhais torácicos; 1-9: músculos. 1: subescapular; 2: coracobraquial; 3: peitorais; 4: grande dorsal; 5 e 6: porções do bíceps braquial; 7: porção longa do deltoide; 8: porção longa do tríceps braquial; 9: braquiorradial; tronco cranial $(\odot)$ tronco caudal $(\square)$; tronco comum $(\star)$; fascículo ventral $(\triangle)$; fascículo dorsal $(\diamond)$; forame supracondilar $(\star)$.

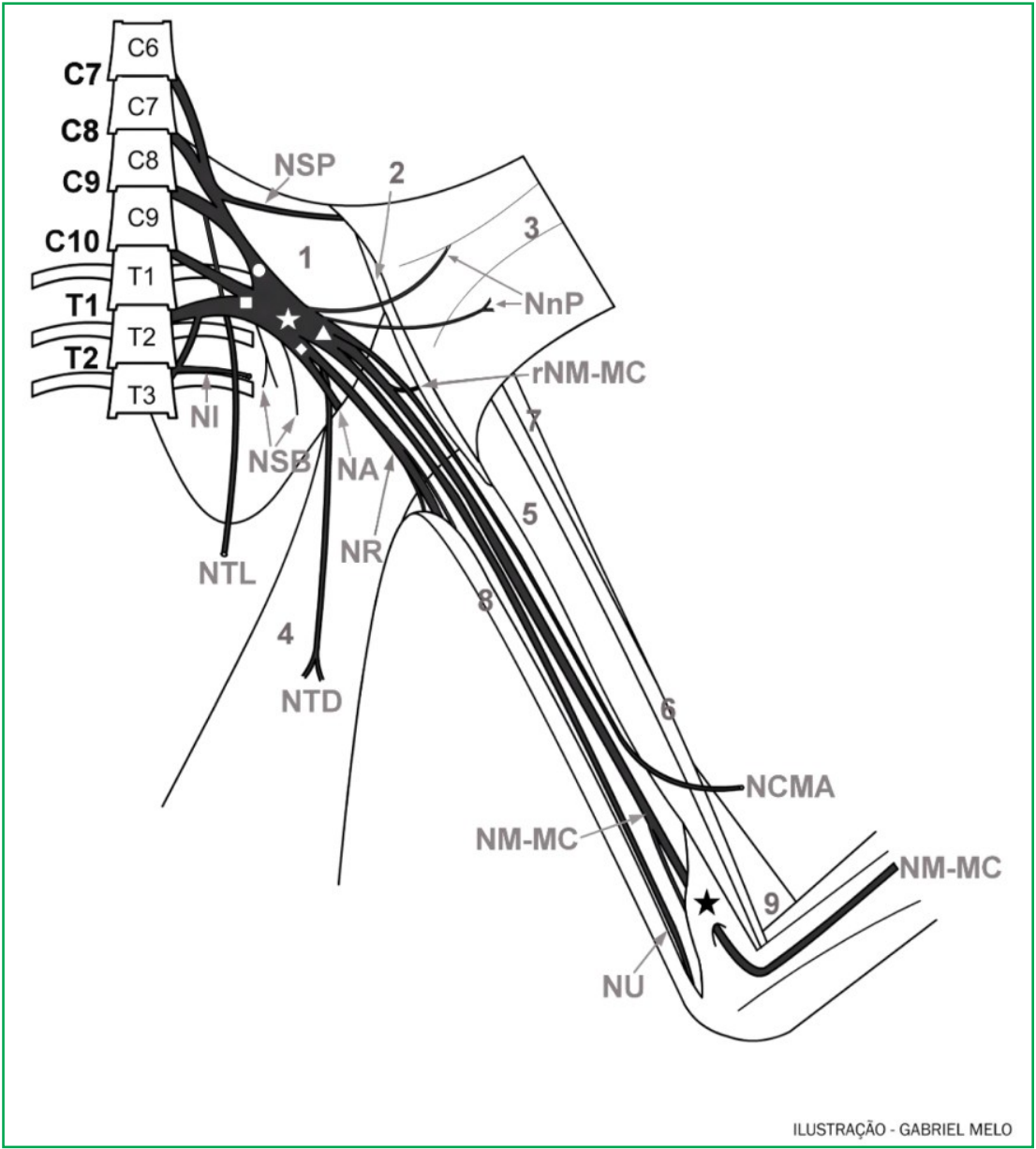


FIGURA 2: (A-D) (A) Vista ventral do plexo braquial de bicho-preguiça-de-coleira (Bradypus torquatus). C6 a C10: sexto ao décimo nervos espinhais cervicais; T1: primeiro nervo espinhal torácico; rC6: ramo comunicante de C6 com C7; ‘〕 : primeira vértebra torácica. Fascículos: ventral (FV) e dorsal (FD); troncos: cranial (TCr) e caudal (TC); * tronco comum. (B) Disposição dos troncos e fascículos. NSP: nervo supraescapular; C9 e C10: nono e décimo nervos espinhais cervicais; T1: primeiro nervo espinhal torácico; troncos: cranial (TCr) e caudal (TC), *: tronco comum; fascículos: ventral (FV) e dorsal (FD). (C) Vista medial do membro torácico. Nervos: cutâneo medial do antebraço (NCMA); ulnar (NU); nervo mediano-musculocutâneo (NM-MC); rNM-MC: ramo do nervo mediano-musculocutâneo; $\star$ : forame supracondilar; Músculos: porção acessória do deltoide (1); porção longa bíceps braquial (2); extensor radial do carpo (3). (D) Vista lateral do membro torácico. nervo radial (NR); veia cefálica (VC); Músculos: porção acessória do músculo deltoide (1); extensor radial do carpo (3); deltoide (4); porção lateral do tríceps braquial (5); braquial (6); peitoral (7); grande dorsal (8).

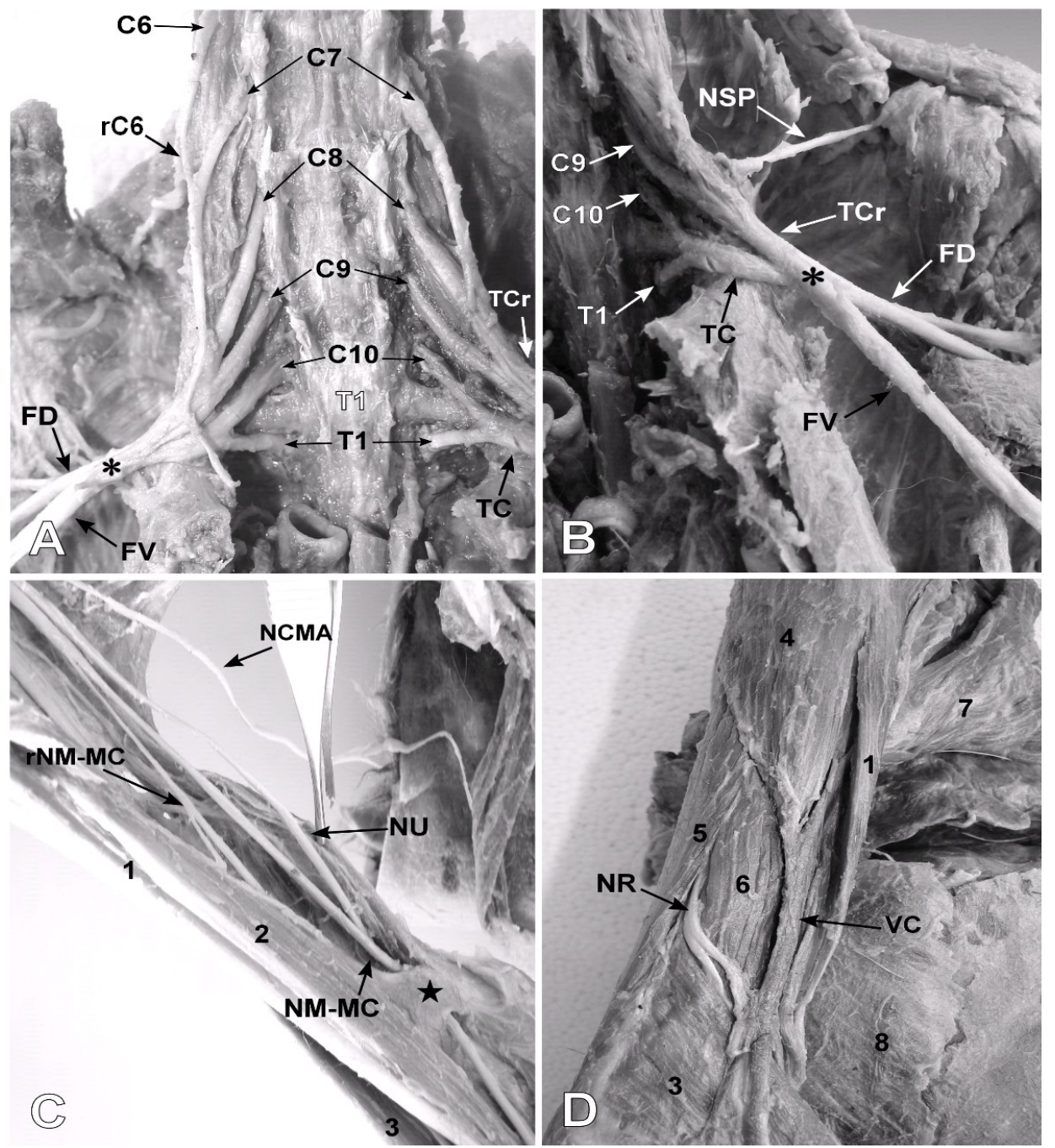

Os nervos musculocutâneo (NMC), mediano (NM), ulnar (NU) e cutâneo medial do antebraço (NCMA) originaram-se do fascículo ventral. O NCMA ramificou-se na pele do antebraço. O NU percorreu o braço sem emitir ramos e seguiu para o antebraço. $\mathrm{O}$ $\mathrm{NM}$ e o NMC seguiram por todo o membro torácico fusionados formando um único nervo, o nervo medianomusculocutâneo (NM-MC), que no braço emitiu ramos para os músculos coracobraquial, bíceps e braquial, além de emitir um ramo para a margem cranial do forame supracondilar do úmero. O NM-MC atravessou o forame com a artéria braquial e seguiu para o antebraço.

Os nervos axilar (NA), toracodorsal (NTD), radial (NR) e subescapulares (NnSB) apresentaram origem comum no fascículo dorsal. O NA passou entre os músculos subescapular e redondo maior e seguiu para a 
face lateral onde emitiu ramos para os músculos redondo maior, deltoide e porção acessória do bíceps, derivada do músculo deltoide.

O NTD ramificou-se no músculo grande dorsal, porém em dois animais emitiu um ramo para o músculo redondo maior. O NR ramificou-se ainda na face medial e emitiu ramos para as três porções do músculo tríceps braquial e para o ancôneo e seguiu para a face lateral emitindo os ramos superficial e profundo que se distribuíram nos músculo do antebraço e mão. Os NnSB foram em número de dois ou três e supriram o músculo subescapular.

\section{Discussão}

A origem do plexo braquial em mamíferos domésticos e silvestres apresenta diferenças na contribuição dos segmentos medulares de acordo com as espécies, gêneros (MELO et al., 2007) e assimetria dos antímeros (FIORETTO et al., 2003), ressaltando a discrepância quando diversas espécies são comparadas. Magilton et al. (1968) relataram a origem dos plexos dos animais domésticos de fazenda com contribuição variando de C6 a T1 em caprinos e ovinos; de C5 a T1 em suíno; de C6 a T2 em equinos e bovinos. Em búfalo (PRASAD; SINHA, 1981), antílope japonês (ATOJI et al., 1987), chinchila (GAMBA et al., 2007) e em veado (MELO et al., 2007) originou-se de C6 a T1; em camelo bactriano de C7 a T1 (WANG 1996); em ornitorrinco e équidna-ouriço de C4 a T2 (KOIZUMI; SAKAI, 1997); de C5 a T2 em babuíno (BOOTH et al., 1997), macaco-prego (RIBEIRO et al., 2005), porco-espinho (AYDIN, 2003) e paca (SCAVONE et al., 2008); de C4 a T1 em capivara (FIORETTO et al., 2003), com origem proveniente de combinações de $\mathbf{C 5}$ a T2 em mocó (SANTANA et al., 2003) e de combinações de C4 a T2 em catetos (MOURA et al., 2007).

Em preguiça-de-bentinho (B. tridactylus) a origem ocorreu de C7 a C10 e T1 a T2 (BIELIK, 1937) e foi a única origem idêntica a de $B$. torquatus, o que pode ser explicado por serem do mesmo gênero e por possuírem nove vértebras cervicais e, consequentemente, dez pares de nervos espinhais cervicais, tornando a origem de seus plexos diferente de todas as espécies de mamíferos e até de outras preguiças (gêneros Choloepus e Bradypus) que também apresentam variação no número de vértebras cervicais (HAUTIER et al., 2010).

Neste estudo, não foi observada variação da origem em relação ao sexo, como em veados (MELO et al., 2007), nem assimetria dos antímeros, como em capivaras (FIORETTO et al., 2003), além de não apresentar ramos comunicantes entre os nervos do plexo, o que divergiu da maioria dos mamíferos (KOIZUMI; SAKAI 1997; GAMBA et al., 2007; MELO et al., 2007; SCAVONE et al., 2008).

O plexo de $B$. torquatus consistiu principalmente de dois troncos e dois fascículos que derivaram os nervos, igualmente ao observado em preguiça-de-bentinho (BIELIK, 1937). Cruz e Adami (2012), ao descreverem a origem do plexo braquial em tamanduá-mirim, espécie da Ordem Xenarthra, na qual também se encontra o bicho-preguiça-de-coleira, descreveram a origem do plexo braquial a partir de três troncos, em contraste com os dois troncos observados em preguiça-de-coleira e em porco-espinho (AYDIN, 2004).

Bielik (1937) ressaltou que existe a probabilidade de contribuição de todas as raízes do plexo (C7 a T2) na união dos troncos cranial e caudal, tornando necessário um estudo mais detalhado para constatar a distribuição das fibras nervosas para a formação dos nervos do plexo. Esta hipótese destaca a importância do conhecimento anatômico do plexo neste gênero para abordagens cirúrgicas, além de ressaltar que traumas ou lesões no nível do tronco comum podem trazer graves problemas, como a paralisia da maior parte do membro.

Os nervos que se originaram antes dos fascículos em Bradypus torquatus diferiram do relatado em mamíferos devido à particularidade do número adicional de segmentos medulares cervicais, porém assemelhou-se a $B$. tridactylus no qual alguns nervos originaram-se antes da formação dos troncos (BIELIK, 1937) (Figura 1).

Os NnP, em preguiça-de-coleira e em porco espinho, originaram-se do tronco caudal e supriram os músculos peitorais (AYDIN, 2003). A origem unisegmentar do NTL - C9 - observada em um exemplar de bicho-preguiça-de-coleira contrastou com a origem plurissegmentar descrita na maioria dos mamíferos, 
embora o território de inervação tenha sido o mesmo, à exceção do descrito em pacas, cuja inervação foi somente o músculo grande dorsal (SCAVONE et al., 2008).

O NSP em preguiça-de-coleira originou-se do tronco cranial ou apenas de $\mathrm{C} 7$ (dois animais), conforme o encontrado em veado e com o mesmo território de inervação, excetuando-se apenas o músculo redondo menor que se fusiona com o músculo infraespinhoso em preguiças (WINDLE; PARSONS, 1899; MELO et al., 2007). Em camelo bactriano (WANG, 1996) e em chinchila (GAMBA et al., 2007), o NSP se formou da parte mais cranial do plexo braquial semelhante a B. torquatus, suprindo os músculos supraespinhoso, infraespinhoso e deltoide.

A origem dos nervos do fascículo ventral na maioria dos animais estudados divergiu do encontrado em B. torquatus, como em animais de fazenda (MAGILTON et al., 1968) e silvestres (MIZUNO, 1969; ATOJI et al., 1987; BOOTH et al., 1997; AYDIN, 2004). Em preguiça-de-bentinho, BIELIK (1937) também observou o surgimento do NM, NU, NMC, os ramos cutâneos do braço e do antebraço a partir do fascículo ventral, porém o NM e o NMC foram tratados como nervos distintos.

Na maior parte dos mamíferos o NM e o NMC têm origem comum, porém são nervos distintos. Somente em antílopes japoneses (ATOJI et al., 1987) e em capivaras (FIORETTO et al., 2003) o NM e o NMC formaram um tronco único em todo o seu trajeto, o NM-MC (nervo mediano musculocutâneo), igual ao observado em preguiça-de-coleira. Em babuínos (BOOTH et al., 1997) e porco-espinho (AYDIN, 2004), a maior parte do NMC seguiu com o NM formando um tronco comum, separando-se apenas no terço distal do braço. Em $B$. torquatus os ramos do tronco NM-NMC mostraram mesma distribuição que em capivaras (FIORETTO et al., 2003), exceto o ramo para o músculo flexor radial do carpo.

O NU, em B. torquatus, não emitiu ramos para o braço e seguiu para o antebraço acordando com os mamíferos em geral. Em chinchilas inervou o antebraço e a mão, porém não emitiu ramos para o braço (GAMBA et al., 2007), como o observado em preguiça-de-coleira.
A origem dos nervos do fascículo dorsal diferiu da maioria dos animais já estudados (MAGILTON et al., 1968; AYDIN, 2004; GAMBA et al., 2007). Entretanto, Bielik (1937) observou em B. tridactylus que o NR e o NA surgiram do fascículo dorsal como o encontrado em B. torquatus, porém não citou o NTD e os NnSB. Atoji et al. (1987) relataram a partir do fascículo dorsal, em antílopes japoneses, o NnSB, NTD, NA, NR e o NSP.

O NA em preguiça-de-coleira assemelhou-se ao encontrado na maioria dos mamíferos, com exceção da inervação dos músculos subescapular (KOIZUMI; SAKAI, 1997; SCAVONE et al., 2008) e braquiocefálico (ATOJI et al., 1987; WANG, 1996; GAMBA et al., 2007), para os quais o nervo axilar não se ramificou em $B$. torquatus.

Em B. torquatus, o NR ramificou-se semelhante ao observado em capivaras (FIORETTO et al. 2003), porém, em chinchilas (GAMBA et al., 2007) e pacas, (SCAVONE et al., 2008) emitiu ramos para o músculo tensor da fáscia do antebraço, ausente no gênero Bradypus (WINDLE; PARSONS, 1899), além suprir os músculos do antebraço. Em babuínos (BOOTH et al., 1997), o NR originou-se de todos os ramos do plexo semelhante à constituição em preguiça-de-coleira.

Apesar de o NTD possuir origem diferente em relação às outras espécies, como capivaras (FIORETTO et al., 2003), veados (MELO et al., 2007) e pacas (SCAVONE et al., 2008), o suprimento foi para o músculo grande dorsal, porém os ramos para o músculo redondo maior aconteceram somente em preguiça-decoleira.

Os $\mathrm{NnSb}$ em capivaras apresentaram mesmo número e inervação ao observado em preguiça, porém sua origem variou em relação ao antímero (FIORETTO et al., 2003). Em porco-espinho (AYDIN, 2004) e chinchilas (GAMBA et al., 2007) supriram os músculos subescapular e redondo maior e em pacas (SCAVONE et al., 2008) ramificou-se somente para o subescapular igualando-se a $B$. torquatus.

O plexo braquial de bicho-preguiça-de-coleira (Bradypus torquatus) derivou-se a partir do $7^{\circ}, 8^{\circ}, 9^{\circ} \mathrm{e}$ $10^{\circ}$ segmentos medulares cervicais e $1^{\circ}$ e $2^{\circ}$ torácicos, que corresponderam aos respectivos nervos espinhais relacionados: C7 a C10 e T1 a T2, sendo a participação de 
T2 variável. A organização anatômica do plexo braquial em troncos e fascículos evidenciou a contribuição da maioria das fibras nervosas dos nervos espinhais relacionados na formação dos nervos periféricos.

\section{Referências}

AMORIM, A. A. J.; AMORIM, M. J. A. A. L.; SILVA, D. R.; PIMENTEL, D. S.; ARAÚJO, F. P.; ALVIM, M. M. S. Origem do plexo braquial no bicho-preguiça (Bradypus variegatus Shinz, 1825). International Journal of Morphology, Temuco, v. 21, n. 1, p. 45, 2003.

ATOJI, Y.; SUZUKI, Y.; SUGIMURA, M. The brachial plexus of the Japanese serow (Capricornis crispus). Anatomischer Anzeiger, Amsterdam, v. 163, n. 1, p. 25-32, 1987.

AYDIN, A. Brachial plexus of the porcupine (Hystrix cristata). Veterinární Medicína, Praga, v. 48, n. 10, p. 301-304, 2003.

AYDIN, A. Nerves originating from brachial plexus in the porcupine (Hystrix cristata). Veterinární Medicína, Praga, v. 49, n. 4, p. 123-128, 2004.

BIELIK, P. Constituição do Plexus Brachialis no Bradypus tridactylus. Arquivos de Anatomia e Antropologia, Lisboa, v. 18, p. 1-4, 1937.

BOOTH, K. K.; BALOYI, F. M.; LUKHELE, O. M. The brachial plexus in the Chacma baboon (Papio ursinus). Journal of Medical Primatology, Copenhagen, v. 26, p. 196-203, 1997.

CHIARELlO, A. G. Diet of the Atlantic forest maned sloth Bradypus torquatus. Journal of Zoology, London, v. 246, p 11-19, 1998.

CHIARELlO, A.; LARA-RUIZ, P.; DA FONSECA, G. A. B.; BERRIDGE, R. IUCN - International Union for Conservation of Nature and Natural Resources. 2011. Disponível em: <http://www. iucnredlist.org/details/3036>. Acesso em: 11 mar. 2012.

CRUZ, G. A. M.; ADAMI, M.; ALMEIDA, A. E. F. S.; SILVA, E. A. A. C.; FARIA, M.M.M. D.; PINTO, M. G. F.; SILVA, R. D. G. Características anatômicas do plexo braquial de tamanduámirim (Tamandua tetradactyla Linnaeus, 1758). Revista Brasileira de Saúde e Produção Animal, v.13, n.3, p.712-719, 2012.

FERNANDEZ, F.; MIRANDA, F. Preguiças: conhecer para preservar. Nosso Clínico, São Paulo, v. 10, n. 58, p. 44-48, 2007.

FIORETTO, E. T.; CASTRO, M. F. S.; GUIDI, W. L.; MAINARDI, R.; SOUZA, R. R.; RIBEIRO, A. A. C. M. Gross anatomic organization of the capybara's (Hydrocharis hydrochaeris) brachial plexus. Anatomia, Histologia, Embryologia, Berlin, v. 32, n. 3, p. 169-174, 2003.

GAMBA, C. O.; CASTRO, T. F.; RICKES, E. M.; MARTINEZ, M. A. P. Sistematização dos territórios nervosos do plexo braquial em chinchila (Chinchilla lanigera). Brazilian Journal of Veterinary Research and Animal Science, São Paulo, v. 44, n. 4, p. 283-289, 2007.

GETTY, R.; SISSON, S.; GROSSMAN, J. D. Anatomia dos animais domésticos. 2 v. 5. ed. Rio de Janeiro: Guanabara Koogan, 1986. 2048 p.

HAUTIER, L.; WEISBECKER, V.; SÁNCHEZ-VILLAGRA, M. R.; GOSWAMI, A.; ASHER, R. J. Skeletal development in sloths and the evolution of mammalian vertebral patterning. Proceedings of the National Academy of Sciences of the United States of America, Washington, v. 107, n. 44, p. 18903-18908, 2010.

INTERNATIONAL COMMITTEE ON VETERINARY GROSS ANATOMICAL NOMENCLATURE. Nomina anatomica veterinaria. 5. ed. Ithaca: Word Association on Veterinary Anatomists, 2005. 190 p.

KOIZUMI, M.; SAKAI, T. On the morphology of the brachial plexus of the platypus (Ornithorhynchus anatinus) and the echidna (Tachyglossus aculeatus). Journal of Anatomy, Maiden, v. 190, p. 447-455, 1997.

MAGILTON, J. H.; GETTY, R.; GHOSHAL, N. G. A comparative morphological study of the brachial plexus of domestic animals (Goat, sheep, ox, pig and horse). Iowa State Journal of Science, Ames, v. 42, p. 245-279, 1968.

MELO, S. R.; GONÇALVES, A. F. N.; SASAHARA, T. H. C.; FIORETTO, E. T.; GERBASI, S. H.; MACHADO, M. R.; GUIMARÃES, G. C.; RIBEIRO, A. A. C. M. Sex-related macrostructural organization of the deer's brachial plexus. Anatomia, Histologia, Embryologia, Berlin, v. 36, n. 4, p. 295-299, 2007.

MENDEL, F. C. Use of hands and feet of three-toed sloths (Bradypus variegatus) during climbing and terrestrial locomotion. Journal of Mammalogy, Lawrence, v. 66, p. 359-366, 1985.

MIZUNO, N. The brachial plexus of a Capuchin Monkey (Cebus capucinus). Primates, Kyoto, v. 10, p. 37-40, 1969.

MOURA, C. E. B.; ALBUQUERQUE, J. F. G.; MAGALHÃES, M. S.; SILVA, N. B.; OLIVEIRA, M. F.; PAPA, P. C. Análise comparativa da origem do plexo braquial de catetos (Tayassu tajacu). Pesquisa Veterinária Brasileira, Seropédica, v. 27, n. 9, p. 357-362, 2007.

PRASAD, J.; SINHA, R. D. Studies on the brachial plexus of Indian buffalo (Bubalus bubalis). Indian Journal of Animal Sciences, Nova Deli v. 51, n. 3, p. 302-307, 1981.

RIBEIRO, A. R.; PRADA, Z. S.; BARROS, R. A. C.; SILVA, D. C. O. Origem do plexo braquial do macaco Cebus apella. Brazilian Journal of Veterinary Research and Animal Science, São Paulo, v. 42, n. 2, p. 143-149, 2005.

SANTANA, J. J.; ALBUQUERQUE, J. F. G.; MOURA, C. E. B.; COSTA, W. P.; OLIVEIRA, M. F.; BARRETO JÚNIOR, R. A.; MIGLINO, M. A. Origem do plexo braquial de mocós (Kerodon rupestris Wied, 1820). Brazilian Journal of Veterinary Research and Animal Science, São Paulo, v. 40, p. 391-396, 2003.

SCAVONE, A. R. F.; MACHADO, M. R. F.; GUIMARÃES, G. C.; OLIVEIRA, F. S.; GERBASI, S. H. B. Análise da origem e distribuição dos nervos periféricos do plexo braquial da paca (Agouti paca, Linnaeus, 1766). Ciência Animal Brasileira, Goiânia, v. 9, n. 4, p. 1046-1055, 2008.

VARELA-LASHERAS, I.; BAKKER, A. J.; MIJE, S. D.; METZ, J. A. J.; ALPHEN, J.; GALIS, F. Breaking evolutionary and pleiotropic constraints in mammals: On sloths, manatees and homeotic mutations. EvoDevo, London, v. 2, n.11, p. 1-27, 2011.

WANG, J. Anatomical study on the formation of the brachial plexus and course and distribution $\mathrm{f}$ its few braches in the Bactrian Camel. Indian Veterinary Journal, Nova Deli, v. 73, p. 169-175, 1996.

WINDLE, B. C. A.; PARSONS, F. G. On the myology of Edentata. Proceedings of the Zoological Society of London, London, v. 1, p. 314-338, 1899. 